\title{
INVERSION FORMULAS INVOLVING ORTHOGONAL POLYNOMIALS AND SOME OF THEIR APPLICATIONS
}

\author{
ROELOF KOEKOEK \\ Delft University of Technology, Faculty of Information Technology and Systems, \\ P.O. Box 5031, 2600 GA Delft, The Netherlands \\ E-mail:koekoek@twi.tudelft.nl
}

\begin{abstract}
We derive inversion formulas involving orthogonal polynomials which can be used to find coefficients of differential equations satisfied by certain generalizations of the classical orthogonal polynomials. As an example we consider special symmetric generalizations of the Hermite polynomials.
\end{abstract}

\section{Introduction}

In $\mathrm{O}$ we found differential equations of spectral type satisfied by the generalized Laguerre polynomials $\left\{L_{n}^{\alpha, M}(x)\right\}_{n=0}^{\infty}$ which are orthogonal on the interval $[0, \infty)$ with respect to the weight function

$$
\frac{1}{\Gamma(\alpha+1)} x^{\alpha} e^{-x}+M \delta(x), \alpha>-1, M \geq 0 .
$$

These orthogonal polynomials were introduced by T.H. Koornwinder in 1 . In order to find the coefficients of these differential equations we had to solve systems of equations of the form

$$
\sum_{i=1}^{\infty} a_{i}(x) D^{i} L_{n}^{(\alpha)}(x)=F_{n}(x), n=1,2,3, \ldots
$$

where $D=\frac{d}{d x}$ denotes the differentiation operator. In 1 H. Bavinck showed that the coefficients $\left\{a_{i}(x)\right\}_{i=1}^{\infty}$ are uniquely determined and can be written in the form

$$
a_{i}(x)=(-1)^{i} \sum_{j=1}^{i} L_{i-j}^{(-\alpha-i-1)}(-x) F_{j}(x), i=1,2,3 \ldots
$$

This result is based on the inversion formula

$$
\sum_{k=j}^{i} L_{i-k}^{(-\alpha-i-1)}(-x) L_{k-j}^{(\alpha+j)}(x)=\delta_{i j}, j \leq i, i, j=0,1,2, \ldots
$$

See also 9 . This inversion formula was derived in a similar way as the inversion formula involving Charlier polynomials found in $\mathrm{b}_{\text {. See also }} \mathrm{a}$ and 
section 3 of this paper. For inversion formulas involving Meixner polynomials the reader is referred to 3 . See also 2. In 41 we used the inversion formula (3) to find differential equations of spectral type satisfied by the Sobolev-type Laguerre polynomials $\left\{L_{n}^{\alpha, M, N}(x)\right\}_{n=0}^{\infty}$ which are othogonal with respect to the Sobolev-type inner product

$$
<f, g>=\frac{1}{\Gamma(\alpha+1)} \int_{0}^{\infty} x^{\alpha} e^{-x} f(x) g(x) d x+M f(0) g(0)+N f^{\prime}(0) g^{\prime}(0),
$$

where $\alpha>-1, M \geq 0$ and $N \geq 0$.

In 15 T.H. Koornwinder also introduced the generalized Jacobi polynomials $\left\{P_{n}^{\alpha, \beta, M, N}(x)\right\}_{n=0}^{\infty}$ which are orthogonal on the interval $[-1,1]$ with respect to the weight function

$$
\frac{\Gamma(\alpha+\beta+2)}{2^{\alpha+\beta+1} \Gamma(\alpha+1) \Gamma(\beta+1)}(1-x)^{\alpha}(1+x)^{\beta}+M \delta(x+1)+N \delta(x-1),
$$

where $\alpha>-1, \beta>-1, M \geq 0$ and $N \geq 0$. In 12 we were looking for differential equations of spectral type satisfied by these generalized Jacobi polynomials. The general case turned out to be very difficult, but in 13 we were able to solve this problem in the special case that $\beta=\alpha$ and $N=M$. In order to find the coefficients of these differential equations we had to solve systems of equations of the form

$$
\sum_{i=1}^{\infty} c_{i}(x) D^{i} P_{n}^{(\alpha, \beta)}(x)=F_{n}(x), n=1,2,3, \ldots
$$

In $\theta$ we showed that the coefficients $\left\{c_{i}(x)\right\}_{i=1}^{\infty}$ are unique and that they can be written in the form

$$
c_{i}(x)=2^{i} \sum_{j=1}^{i} \frac{\alpha+\beta+2 j+1}{(\alpha+\beta+j+1)_{i+1}} P_{i-j}^{(-\alpha-i-1,-\beta-i-1)}(x) F_{j}(x), i=1,2,3 \ldots
$$

This result is based on the inversion formula

$$
\begin{aligned}
& \sum_{k=j}^{i} \frac{\alpha+\beta+2 k+1}{(\alpha+\beta+k+j+1)_{i-j+1}} \\
& \times P_{i-k}^{(-\alpha-i-1,-\beta-i-1)}(x) P_{k-j}^{(\alpha+j, \beta+j)}(x)=\delta_{i j}, j \leq i, i, j=0,1,2, \ldots,
\end{aligned}
$$

which is proved in 6 . This inversion formula was derived in a completely different way than the inversion formulas mentioned before. In $\mathrm{g}$ it is shown that this inversion formula (with $\beta=\alpha$ ) can be used to derive the results obtained in 13 in an easier way. Finally in 10 this inversion formula is used to solve the problem for all $\alpha>-1, \beta>-1, M \geq 0$ and $N \geq 0$. 
In this paper we will derive several kinds of inversion formulas and we will show how they can be applied to find coefficients of differential equations for generalizations of some classical orthogonal polynomials.

\section{Some classical orthogonal polynomials}

In this section we will recall some formulas involving classical orthogonal polynomials which we will use in this paper. For details the reader is referred to 14 .

The Meixner-Pollaczek polynomials $\left\{P_{n}^{(\lambda)}(x ; \phi)\right\}_{n=0}^{\infty}$ can be defined by their generating function

$$
\left(1-e^{i \phi} t\right)^{-\lambda+i x}\left(1-e^{-i \phi} t\right)^{-\lambda-i x}=\sum_{n=0}^{\infty} P_{n}^{(\lambda)}(x ; \phi) t^{n} .
$$

The classical Jacobi polynomials $\left\{P_{n}^{(\alpha, \beta)}(x)\right\}_{n=0}^{\infty}$ can be defined for all $\alpha$ and $\beta$ and $n \in\{0,1,2, \ldots\}$ by

$$
P_{n}^{(\alpha, \beta)}(x)=\sum_{k=0}^{n} \frac{(n+\alpha+\beta+1)_{k}}{k !} \frac{(\alpha+k+1)_{n-k}}{(n-k) !}\left(\frac{x-1}{2}\right)^{k} .
$$

They satisfy the orthogonality relation

$$
\begin{array}{r}
\frac{\Gamma(\alpha+\beta+2)}{2^{\alpha+\beta+1} \Gamma(\alpha+1) \Gamma(\beta+1)} \int_{-1}^{1}(1-x)^{\alpha}(1+x)^{\beta} P_{m}^{(\alpha, \beta)}(x) P_{n}^{(\alpha, \beta)}(x) d x \\
\quad=\frac{\alpha+\beta+1}{2 n+\alpha+\beta+1} \frac{(\alpha+1)_{n}(\beta+1)_{n}}{(\alpha+\beta+1)_{n} n !} \delta_{m n}, m, n=0,1,2, \ldots
\end{array}
$$

The Gegenbauer or ultraspherical polynomials $\left\{G_{n}^{(\lambda)}(x)\right\}_{n=0}^{\infty}$ form a special case of the classical Jacobi polynomials. In fact we have

$$
G_{n}^{(\lambda)}(x)=\frac{(2 \lambda)_{n}}{\left(\lambda+\frac{1}{2}\right)_{n}} P_{n}^{\left(\lambda-\frac{1}{2}, \lambda-\frac{1}{2}\right)}(x), \lambda>-\frac{1}{2}, \lambda \neq 0 .
$$

These ultraspherical polynomials can also be defined by their generating function

$$
\left(1-2 x t+t^{2}\right)^{-\lambda}=\sum_{n=0}^{\infty} G_{n}^{(\lambda)}(x) t^{n}
$$


The special case $\lambda=0$ needs another normalization. In that case we have the Chebyshev polynomials of the first kind $\left\{T_{n}(x)\right\}_{n=0}^{\infty}$ given by

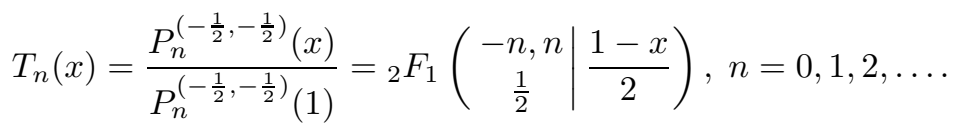

Their generating function equals

$$
\frac{1-x t}{1-2 x t+t^{2}}=\sum_{n=0}^{\infty} T_{n}(x) t^{n}
$$

The Chebyshev polynomials of the second kind $\left\{U_{n}(x)\right\}_{n=0}^{\infty}$ are given by

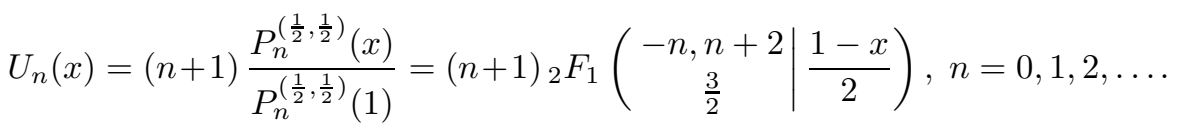

These polynomials can also be defined by their generating function

$$
\frac{1}{1-2 x t+t^{2}}=\sum_{n=0}^{\infty} U_{n}(x) t^{n}
$$

Finally the classical Legendre (or spherical) polynomials $\left\{P_{n}(x)\right\}_{n=0}^{\infty}$ form another special case of the classical Jacobi polynomials. In fact we have

$$
P_{n}(x)=P_{n}^{(0,0)}(x)=\sum_{k=0}^{n} \frac{(n+k) !}{(n-k) !(k !)^{2}}\left(\frac{x-1}{2}\right)^{k}, n=0,1,2, \ldots
$$

These Legendre polynomials can also be defined by their generating function

$$
\frac{1}{\sqrt{1-2 x t+t^{2}}}=\sum_{n=0}^{\infty} P_{n}(x) t^{n} .
$$

Note that the Legendre polynomials also form a special case of the ultraspherical polynomials, since we have

$$
P_{n}(x)=G_{n}^{\left(\frac{1}{2}\right)}(x), n=0,1,2, \ldots
$$

The classical Laguerre polynomials $\left\{L_{n}^{(\alpha)}(x)\right\}_{n=0}^{\infty}$ can be defined for all $\alpha$ and $n \in\{0,1,2, \ldots\}$ as

$$
L_{n}^{(\alpha)}(x)=\sum_{k=0}^{n}(-1)^{k}\left(\begin{array}{c}
n+\alpha \\
n-k
\end{array}\right) \frac{x^{k}}{k !}=\sum_{k=0}^{n}(-1)^{k} \frac{(\alpha+k+1)_{n-k}}{(n-k) !} \frac{x^{k}}{k !} .
$$

inversion: submitted to World Scientific on June 10, 1999 
The generating function for the classical Laguerre polynomials is given by

$$
(1-t)^{-\alpha-1} \exp \left(\frac{x t}{t-1}\right)=\sum_{n=0}^{\infty} L_{n}^{(\alpha)}(x) t^{n}
$$

Further we have for $n=0,1,2, \ldots$

$$
D^{i} L_{n}^{(\alpha)}(x)=(-1)^{i} L_{n-i}^{(\alpha+i)}(x), i=0,1,2, \ldots, n .
$$

Another family of continuous orthogonal polynomials is the one named after Hermite. The classical Hermite polynomials $\left\{H_{n}(x)\right\}_{n=0}^{\infty}$ can be defined by their generating function

$$
\exp \left(x t-\frac{1}{4} t^{2}\right)=\sum_{n=0}^{\infty} H_{n}(x) t^{n} .
$$

Here we used another normalization than in 14 . This one turns out to be more convenient in this paper. These classical Hermite polynomials satisfy the orthogonality relation

$$
\frac{1}{\sqrt{\pi}} \int_{-\infty}^{\infty} e^{-x^{2}} H_{m}(x) H_{n}(x) d x=\frac{\delta_{m n}}{2^{n} n !}, m, n=0,1,2, \ldots
$$

Further we have for $n=0,1,2, \ldots$

$$
D^{i} H_{n}(x)=H_{n-i}(x), i=0,1,2, \ldots, n .
$$

From the generating function it follows that

$$
H_{2 n+1}(0)=0 \text { and } H_{2 n}(0)=\frac{(-1)^{n}}{2^{2 n} n !}, n=0,1,2, \ldots
$$

Further we will use the kernels

$$
K_{n}(x, y)=\sum_{k=0}^{n} 2^{k} k ! H_{k}(x) H_{k}(y), n=0,1,2, \ldots .
$$

By using (17) we easily find that for $n=0,1,2, \ldots$

$$
K_{2 n+1}(x, 0)=K_{2 n}(x, 0)=\sum_{k=0}^{n}(-1)^{k} \frac{(2 k) !}{k !} H_{2 k}(x)
$$

and

$$
K_{2 n+1}(0,0)=K_{2 n}(0,0)=\sum_{k=0}^{n} \frac{(2 k) !}{2^{2 k}(k !)^{2}}=\sum_{k=0}^{n} \frac{\left(\frac{1}{2}\right)_{k}}{k !}=\frac{\left(\frac{3}{2}\right)_{n}}{n !} .
$$


Finally we will consider the discrete orthogonal polynomials named after Meixner and Charlier. We choose normalizations different from those in 14 . The classical Meixner polynomials $\left\{M_{n}^{(\beta)}(x ; c)\right\}_{n=0}^{\infty}$ can be defined by their generating function

$$
\left(1-\frac{t}{c}\right)^{x}(1-t)^{-x-\beta}=\sum_{n=0}^{\infty} M_{n}^{(\beta)}(x ; c) t^{n} .
$$

The Meixner polynomials are connected to the classical Jacobi polynomials in the following way

$$
M_{n}^{(\beta)}(x ; c)=P_{n}^{(\beta-1,-n-\beta-x)}\left(\frac{2-c}{c}\right), n=0,1,2, \ldots
$$

The classical Charlier polynomials $\left\{C_{n}^{(a)}(x)\right\}_{n=0}^{\infty}$ can also be defined by their generating function

$$
e^{-a t}(1+t)^{x}=\sum_{n=0}^{\infty} C_{n}^{(a)}(x) t^{n}
$$

\section{Some inversion formulas}

In $⿴$ 年 we observed that the generating function (23) implies that

$$
1=e^{-a t}(1+t)^{x} e^{a t}(1+t)^{-x}=\sum_{n=0}^{\infty}\left(\sum_{k=0}^{n} C_{k}^{(a)}(x) C_{n-k}^{(-a)}(-x)\right) t^{n},
$$

which implies that

$$
\sum_{k=0}^{n} C_{k}^{(a)}(x) C_{n-k}^{(-a)}(-x)=\left\{\begin{array}{l}
1, n=0 \\
0, n=1,2,3 \ldots
\end{array}\right.
$$

or

$$
\sum_{k=j}^{i} C_{i-k}^{(-a)}(-x) C_{k-j}^{(a)}(x)=\delta_{i j}, j \leq i, i, j=0,1,2, \ldots
$$

As already indicated in 9 this formula (24) can be interpreted as follows. If we define the matrix $T=\left(t_{i j}\right)_{i, j=0}^{n}$ with entries

$$
t_{i j}= \begin{cases}C_{i-j}^{(a)}(x), & j \leq i \\ 0, & j>i\end{cases}
$$


then this matrix $T$ is a triangular matrix with determinant 1 and the inverse $U$ of $T$ is given by $T^{-1}=U=\left(u_{i j}\right)_{i, j=0}^{n}$ with entries

$$
u_{i j}= \begin{cases}C_{i-j}^{(-a)}(-x), & j \leq i \\ 0, & j>i .\end{cases}
$$

Therefore we call (24) an inversion formula.

In the same way we find from the generating function (13) for the classical Laguerre polynomials

$$
\sum_{k=j}^{i} L_{i-k}^{(-\alpha-2)}(-x) L_{k-j}^{(\alpha)}(x)=\delta_{i j}, j \leq i, i, j=0,1,2, \ldots
$$

However, in view of (14) this inversion formula cannot be used to solve the systems of the equations of the form (1). In $1 \mathrm{H}$. Bavinck observed that it also follows from the generating function (13) that

$$
\begin{aligned}
(1-t)^{i-j-1} & =(1-t)^{-\alpha-j-1} \exp \left(\frac{x t}{t-1}\right)(1-t)^{\alpha+i} \exp \left(\frac{-x t}{t-1}\right) \\
& =\sum_{n=0}^{\infty}\left(\sum_{k=0}^{n} L_{k}^{(\alpha+j)}(x) L_{n-k}^{(-\alpha-i-1)}(-x)\right) t^{n}
\end{aligned}
$$

which implies, by comparing the coefficients of $t^{i-j}$ on both sides, that

$$
\sum_{k=0}^{i-j} L_{k}^{(\alpha+j)}(x) L_{i-j-k}^{(-\alpha-i-1)}(-x)=\delta_{i j}, j \leq i, i, j=0,1,2, \ldots,
$$

which is equivalent to (3). This inversion formula implies that the system of equations (1) has the unique solution given by (2).

\section{More (inversion) formulas}

Applying the method described in the preceding section to the generating function (10) for the Chebyshev polynomials of the second kind and the generating function (11) for the Legendre polynomials we obtain

$$
\begin{aligned}
\sum_{n=0}^{\infty} U_{n}(x) t^{n} & =\frac{1}{1-2 x t+t^{2}}=\frac{1}{\sqrt{1-2 x t+t^{2}}} \frac{1}{\sqrt{1-2 x t+t^{2}}} \\
& =\sum_{k=0}^{\infty} P_{k}(x) t^{k} \sum_{m=0}^{\infty} P_{m}(x) t^{m}=\sum_{n=0}^{\infty}\left(\sum_{k=0}^{n} P_{k}(x) P_{n-k}(x)\right) t^{n},
\end{aligned}
$$

inversion: submitted to World Scientific on June 10, 1999 
which implies that

$$
\sum_{k=0}^{n} P_{k}(x) P_{n-k}(x)=U_{n}(x), n=0,1,2, \ldots
$$

Another interesting formula of this kind can be found by using the generating function (10) for the Chebyshev polynomials of the second kind and the generating function (91) for the Chebyshev polynomials of the first kind. In fact, we have

$$
\begin{aligned}
\sum_{n=0}^{\infty} U_{n}(x) t^{n} & =\frac{1}{1-2 x t+t^{2}}=\frac{1}{1-x t} \frac{1-x t}{\sqrt{1-2 x t+t^{2}}} \\
& =\sum_{k=0}^{\infty} x^{k} t^{k} \sum_{m=0}^{\infty} T_{m}(x) t^{m}=\sum_{n=0}^{\infty}\left(\sum_{k=0}^{n} x^{k} T_{n-k}(x)\right) t^{n}
\end{aligned}
$$

which implies that

$$
\sum_{k=0}^{n} x^{k} T_{n-k}(x)=U_{n}(x), n=0,1,2, \ldots
$$

As before we can use the generating function (8) for the ultraspherical polynomials to obtain the inversion formula

$$
\sum_{k=j}^{i} G_{i-k}^{(-\lambda)}(x) G_{k-j}^{(\lambda)}(x)=\delta_{i j}, j \leq i, i, j=0,1,2, \ldots
$$

In view of (12) the special (limit) case $\lambda=\frac{1}{2}$ should lead to an inversion formula for the Legendre polynomials. If we define for every positive integer $N$ the matrix $A=\left(a_{i j}\right)_{i, j=1}^{N}$ with entries

$$
a_{i j}= \begin{cases}P_{i-j}(x) & , j \leq i \\ 0, & j>i,\end{cases}
$$

then this matrix is a triangular matrix with determinant 1 and hence invertible. Now we have $G_{0}^{(\lambda)}(x)=1, G_{1}^{(\lambda)}(x)=2 \lambda x \rightarrow-x$ for $\lambda \rightarrow-\frac{1}{2}$ and for $n=2,3,4, \ldots$

$$
G_{n}^{(\lambda)}(x)=\frac{(2 \lambda)_{n}}{\left(\lambda+\frac{1}{2}\right)_{n}} P_{n}^{\left(\lambda-\frac{1}{2}, \lambda-\frac{1}{2}\right)}(x) \rightarrow \frac{-2}{n-1} P_{n}^{(-1,-1)}(x) \text { for } \lambda \rightarrow-\frac{1}{2} .
$$

Now we have by using (6) for $n=2,3,4, \ldots$.

$$
B_{n}(x):=\frac{-2}{n-1} P_{n}^{(-1,-1)}(x)=\frac{1}{n}(1-x) P_{n-1}^{(1,-1)}(x) .
$$


Hence, the inverse $A^{-1}=B=\left(b_{i j}\right)_{i, j=1}^{N}$ is given by

$$
b_{i j}= \begin{cases}0, & i<j \\ 1, & i=j \\ -x & i=j+1 \\ B_{i-j}(x), & i \geq j+2 .\end{cases}
$$

In case of the Chebyshev polynomials of the second kind we can obtain an inversion formula as follows. If we define for every positive integer $N$ the matrix $A=\left(a_{i j}\right)_{i, j=1}^{N}$ with entries

$$
a_{i j}= \begin{cases}U_{i-j}(x) & , j \leq i \\ 0, & j>i\end{cases}
$$

then this matrix is a triangular matrix with determinant 1 and hence invertible. It is not difficult to show that its inverse $A^{-1}=B=\left(b_{i j}\right)_{i, j=1}^{N}$ is given by

$$
b_{i j}= \begin{cases}1, & i=j \\ -2 x, & i=j+1 \\ 1, & i=j+2 \\ 0, & \text { otherwise. }\end{cases}
$$

This can be shown by writing

$$
B A=C=\left(c_{i j}\right)_{i, j=1}^{N} \text { with } c_{i j}=\sum_{k=1}^{N} b_{i k} a_{k j}
$$

and showing that $C=I$, the identity matrix. This is done by using the well-known relation

$$
U_{n}(x)-2 x U_{n+1}(x)+U_{n+2}(x)=0, n=0,1,2, \ldots .
$$

In case of the Chebyshev polynomials of the first kind we consider the matrix $A=\left(a_{i j}\right)_{i, j=1}^{N}$ for every positive integer $N$ with entries

$$
a_{i j}= \begin{cases}T_{i-j}(x), & j \leq i \\ 0, & j>i\end{cases}
$$

Then this matrix is also a triangular matrix with determinant 1 and hence invertible. The inverse $A^{-1}=B=\left(b_{i j}\right)_{i, j=1}^{N}$ is given by

$$
b_{i j}= \begin{cases}0, & i<j \\ 1, & i=j \\ -x, & i=j+1 \\ x^{i-j-2}\left(1-x^{2}\right) & i \geq j+2 .\end{cases}
$$

inversion: submitted to World Scientific on June 10, 1999 
This can also be shown by writing

$$
B A=C=\left(c_{i j}\right)_{i, j=1}^{N} \text { with } c_{i j}=\sum_{k=1}^{N} b_{i k} a_{k j}
$$

and showing that $C=I$, the identity matrix. This is done by using the formula 25) and the well-known relation

$$
\left(1-x^{2}\right) U_{n}(x)-x T_{n+1}(x)+T_{n+2}(x)=0, n=0,1,2, \ldots .
$$

The generating function (5) can also be used to find inversion formulas involving Meixner-Pollaczek polynomials. In fact we have

$$
\sum_{k=j}^{i} P_{i-k}^{(-\lambda)}(-x ; \phi) P_{k-j}^{(\lambda)}(x ; \phi)=\delta_{i j}, j \leq i, i, j=0,1,2, \ldots
$$

or

$$
\sum_{k=j}^{i} P_{i-k}^{(-\lambda)}(x ;-\phi) P_{k-j}^{(\lambda)}(x ; \phi)=\delta_{i j}, j \leq i, i, j=0,1,2, \ldots
$$

By using the generating function (21) for the Meixner polynomials we find the inversion formula

$$
\sum_{k=j}^{i} M_{i-k}^{(-\beta)}(-x ; c) M_{k-j}^{(\beta)}(x ; c)=\delta_{i j}, j \leq i, i, j=0,1,2, \ldots
$$

We remark that this inversion formula is different from the one obtained in $\mathrm{B}$. See also 2 for an application of that inversion formula.

Note that the generating function (15) for the classical Hermite polynomials implies that

$$
1=\exp \left(x t-\frac{1}{4} t^{2}\right) \exp \left(-x t+\frac{1}{4} t^{2}\right)=\sum_{n=0}^{\infty}\left(\sum_{k=0}^{n} H_{k}(x) H_{n-k}(i x) i^{n-k}\right) t^{n},
$$

which implies that

$$
\sum_{k=0}^{n} H_{k}(x) H_{n-k}(i x) i^{n-k}=\left\{\begin{array}{l}
1, n=0 \\
0, n=1,2,3 \ldots
\end{array}\right.
$$

In view of (16) this formula can be used as follows. A system of equations of the form

$$
F_{n}(x)=\sum_{k=1}^{\infty} a_{k}(x) D^{k} H_{n}(x), n=1,2,3, \ldots,
$$


where the coefficients $\left\{a_{k}(x)\right\}_{k=1}^{\infty}$ are polynomials which are independent of $n$, has the unique solution

$$
a_{k}(x)=\sum_{j=1}^{k} i^{k-j} H_{k-j}(i x) F_{j}(x), k=1,2,3, \ldots
$$

\section{Inversion formulas involving Jacobi polynomials}

In 9 we have found the inversion formula (位) involving Jacobi polynomials. As mentioned before this formula was found in a completely different way. The well-known generating function for the classical Jacobi polynomials has a different structure, which implies that the method used before cannot be used in that case. In 9 we proved that for $n=0,1,2, \ldots$ we have

$$
\sum_{k=0}^{n} \frac{\alpha+\beta+2 k+1}{(\alpha+\beta+k+1)_{n+1}} P_{k}^{(\alpha, \beta)}(x) P_{n-k}^{(-n-\alpha-1,-n-\beta-1)}(y)=\frac{1}{n !}\left(\frac{x-y}{2}\right)^{n} .
$$

Now $y=x$ leads to the inversion formula (田). If $y=-x$ this leads to a formula which was used in $\beta$ in the case that $\beta=\alpha$.

By using the relation (22) between the Meixner and the Jacobi polynomials we find from the inversion formula (28) for the Meixner polynomials that

$$
\sum_{k=j}^{i} P_{i-k}^{(-\alpha-2,-\beta-i+k)}(x) P_{k-j}^{(\alpha, \beta-k+j)}(x)=\delta_{i j}, j \leq i, i, j=0,1,2, \ldots
$$

Another inversion formula involving Jacobi polynomials can be obtained from the inversion formula (26) for the ultraspherical polynomials. By using (7) and after setting $\lambda=\alpha+\frac{1}{2}$ this leads to

$$
\begin{aligned}
& \sum_{k=j}^{i} \frac{(-2 \alpha-1)_{i-k}}{(-\alpha)_{i-k}} \frac{(2 \alpha+1)_{k-j}}{(\alpha+1)_{k-j}} \\
& \quad \times P_{i-k}^{(-\alpha-1,-\alpha-1)}(x) P_{k-j}^{(\alpha, \alpha)}(x)=\delta_{i j}, j \leq i, i, j=0,1,2, \ldots
\end{aligned}
$$

\section{Applications to differential equations}

In this section we will investigate the generalized Hermite polynomials $\left\{H_{n}^{M}(x)\right\}_{n=0}^{\infty}$ which are orthogonal on the real line with respect to the weight function

$$
w(x)=\frac{1}{\sqrt{\pi}} e^{-x^{2}}+M \delta(x), M \geq 0 .
$$

inversion: submitted to World Scientific on June 10, 1999 
In 6 these generalized Hermite polynomials are called special (linear) perturbations of the classical Hermite polynomials. They can be represented in terms of the kernels (18) as (see 4 )

$$
H_{n}^{M}(x)=H_{n}(x)+M Q_{n}(x), n=0,1,2, \ldots,
$$

where $Q_{0}(x)=0$ and

$$
Q_{n}(x)=\left|\begin{array}{cc}
H_{n}(x) & K_{n-1}(x, 0) \\
H_{n}(0) & K_{n-1}(0,0)
\end{array}\right|=\sum_{k=0}^{n} q_{n, k} H_{k}(x), n=1,2,3, \ldots
$$

with, by using (19), for $n=1,2,3, \ldots$

$$
q_{n, n}=K_{n-1}(0,0) \text { and } q_{n, k}=-2^{k} k ! H_{k}(0) H_{n}(0), k=0,1,2, \ldots, n-1 .
$$

In 1 it is shown that these generalized Hermite polynomials satisfy a differential equation of the form

$$
M \sum_{k=1}^{\infty} a_{k}(x) y^{(k)}(x)+y^{\prime \prime}(x)-2 x y^{\prime}(x)+\left(2 n+M \alpha_{n}\right) y(x)=0,
$$

where the coefficients $\left\{a_{k}(x)\right\}_{k=1}^{\infty}$ are polynomials with degree $\left[a_{k}(x)\right] \leq k, k=$ $1,2,3, \ldots$ which are independent of $n$. Moreover it is shown that the 'eigenvalue' parameters $\left\{\alpha_{2 n+1}\right\}_{n=0}^{\infty}$ can be chosen arbitrarily,

$$
\alpha_{0}=0 \text { and } \alpha_{2 n}=\sum_{j=1}^{n}\left(\lambda_{2 j}-\lambda_{2 j-2}\right) q_{2 j, 2 j}, n=1,2,3 \ldots,
$$

where $\lambda_{n}=2 n, n=0,1,2, \ldots$ Hence, $\lambda_{2 j}-\lambda_{2 j-2}=4, j=1,2,3, \ldots$ and by using (20)

$$
q_{2 j, 2 j}=K_{2 j-1}(0,0)=\frac{\left(\frac{3}{2}\right)_{j-1}}{(j-1) !}, j=1,2,3, \ldots
$$

This implies that

$$
\alpha_{2 n}=4 \sum_{j=1}^{n} \frac{\left(\frac{3}{2}\right)_{j-1}}{(j-1) !}=4 \sum_{k=0}^{n-1} \frac{\left(\frac{3}{2}\right)_{k}}{k !}=4 \frac{\left(\frac{5}{2}\right)_{n-1}}{(n-1) !}, n=1,2,3, \ldots
$$

In order to find the coefficients $\left\{a_{k}(x)\right\}_{k=1}^{\infty}$ we set $y(x)=H_{n}^{M}(x)=H_{n}(x)+$ $M Q_{n}(x)$ in the differential equation (31) and view the left-hand side as a polynomial in $M$. Then the coefficients of this polynomial must vanish, hence $\sum_{k=1}^{\infty} a_{k}(x) D^{k} H_{n}(x)=-\alpha_{n} H_{n}(x)-Q_{n}^{\prime \prime}(x)+2 x Q_{n}^{\prime}(x)-2 n Q_{n}(x), n=0,1,2, \ldots$ 
and

$$
\sum_{k=1}^{\infty} a_{k}(x) D^{k} Q_{n}(x)=-\alpha_{n} Q_{n}(x), n=0,1,2, \ldots
$$

Since we have, by using (17) and (20),

$$
Q_{2 n+1}(x)=K_{2 n}(0,0) H_{2 n+1}(x)=\frac{\left(\frac{3}{2}\right)_{n}}{n !} H_{2 n+1}(x), n=0,1,2, \ldots
$$

and

$$
\frac{\left(\frac{3}{2}\right)_{n}}{n !} \neq 0, n=0,1,2, \ldots
$$

both systems of equations lead to

$$
\sum_{k=1}^{2 n+1} a_{k}(x) H_{2 n+1-k}(x)=-\alpha_{2 n+1} H_{2 n+1}(x), n=0,1,2, \ldots
$$

Further we have

$$
Q_{2 n}(x)=\sum_{k=0}^{n} q_{2 n, 2 k} H_{2 k}(x), n=1,2,3, \ldots,
$$

where, by using (20),

$$
q_{2 n, 2 n}=K_{2 n-1}(0,0)=\frac{\left(\frac{3}{2}\right)_{n-1}}{(n-1) !}, n=1,2,3, \ldots
$$

and, by using (17), for $k=0,1,2, \ldots, n-1$ and $n=1,2,3, \ldots$

$$
q_{2 n, 2 k}=-2^{2 k}(2 k) ! H_{2 k}(0) H_{2 n}(0)=\frac{(-1)^{n+k+1}(2 k) !}{2^{2 n} n ! k !} .
$$

Now we have for $n=1,2,3, \ldots$

$$
\begin{aligned}
Q_{2 n}^{\prime \prime}(x)-2 x Q_{2 n}^{\prime}(x) & =\sum_{k=0}^{n} q_{2 n, 2 k}\left[H_{2 k}^{\prime \prime}(x)-2 x H_{2 k}^{\prime}(x)\right] \\
& =\sum_{k=0}^{n} q_{2 n, 2 k}\left[-4 k H_{2 k}(x)\right] .
\end{aligned}
$$

Hence, for $n=1,2,3, \ldots$ we obtain

$$
\begin{array}{r}
-\alpha_{2 n} H_{2 n}(x)-Q_{2 n}^{\prime \prime}(x)+2 x Q_{2 n}^{\prime}(x)-4 n Q_{2 n}(x) \\
=-\alpha_{2 n} H_{2 n}(x)-4 \sum_{k=0}^{n}(n-k) q_{2 n, 2 k} H_{2 k}(x),
\end{array}
$$


which leads for $n=1,2,3, \ldots$ to

$$
\sum_{k=1}^{2 n} a_{k}(x) H_{2 n-k}(x)=-\alpha_{2 n} H_{2 n}(x)-4 \sum_{k=0}^{n}(n-k) q_{2 n, 2 k} H_{2 k}(x) .
$$

Hence, with (32) and (33) we have found that

$$
\sum_{k=1}^{\infty} a_{k}(x) D^{k} H_{n}(x)=\sum_{k=1}^{n} a_{k}(x) H_{n-k}(x)=F_{n}(x), n=1,2,3, \ldots
$$

where

$$
\left\{\begin{array}{l}
F_{2 n+1}(x)=-\alpha_{2 n+1} H_{2 n+1}(x), n=0,1,2, \ldots \\
F_{2 n}(x)=-\alpha_{2 n} H_{2 n}(x)-4 \sum_{k=0}^{n}(n-k) q_{2 n, 2 k} H_{2 k}(x), n=1,2,3, \ldots
\end{array}\right.
$$

This system of equations has the form (29). So we can use (30) to conclude that

$$
a_{k}(x)=\sum_{j=1}^{k} i^{k-j} H_{k-j}(i x) F_{j}(x), k=1,2,3, \ldots
$$

We emphasize that these generalized Hermite polynomials are orthogonal with respect to a weight function consisting of the classical Hermite weight function and a Dirac delta distribution at the origin. Therefore these generalized Hermite polynomials could be considered as Krall-Hermite polynomials, but these are quite different from the Krall-Hermite polynomials considered in 6 which are not orthogonal.

Finally, in 16 it is shown that these generalized Hermite polynomials cannot satisfy a finite order differential equation of the form (31).

\section{References}

1. H. BAVINCK : A direct approach to Koekoek's differential equation for generalized Laguerre polynomials. Acta Math. Hungar. 66, 1995, 247253.

2. H. BAVINCK : Generalizations of Meixner polynomials which are eigenfunctions of a difference operator. J. Differ. Equations Appl. 5, 1999, 143-153.

3. H. BAVINCK \& H. VAN HAERINGEN : Difference equations for generalized Meixner polynomials. J. Math. Anal. Appl. 184, 1994, 453-463.

inversion: submitted to World Scientific on June 10, 1999 
4. H. BAvinck \& J. Koekoek : Differential operators having symmetric orthogonal polynomials as eigenfunctions. J. Comput. Appl. Math., to appear.

5. H. BAVINCK \& R. KoEKoek : On a difference equation for generalizations of Charlier polynomials. J. Approx. Theory 81, 1995, 195-206.

6. F.A. Grünbaum, L. Haine \& E. Horozov : On the Krall-Hermite and the Krall-Bessel polynomials. Internat. Math. Res. Notices 19, 1997, 953-966.

7. J. Koekoek \& R. Koekoek : On a differential equation for Koornwinder's generalized Laguerre polynomials. Proc. Amer. Math. Soc. 112, 1991, 1045-1054.

8. J. Kоекоек \& R. Kоекоек : Finding differential equations for symmetric generalized ultraspherical polynomials by using inversion methods. International Workshop on Orthogonal Polynomials in Mathematical Physics (Leganés, 1996), Univ. Carlos III de Madrid, Leganés, 1997, 103-111.

9. J. Koekoek \& R. Koekoek : The Jacobi inversion formula. Complex Variables, to appear.

10. J. Ковкоек \& R. Kоєкоек : Differential equations for generalized Jacobi polynomials. Submitted for publication.

11. J. Koекоек, R. Kоекоек \& H. Bavinck : On differential equations for Sobolev-type Laguerre polynomials. Trans. Amer. Math. Soc. 350, 1998, 347-393.

12. R. KоеKOEK : The search for differential equations for certain sets of orthogonal polynomials. J. Comput. Appl. Math. 49, 1993, 111-119.

13. R. Kоекоек : Differential equations for symmetric generalized ultraspherical polynomials. Trans. Amer. Math. Soc. 345, 1994, 47-72.

14. R. Koekoek \& R.F. Swarttouw : The Askey-scheme of hypergeometric orthogonal polynomials and its q-analogue. Delft University of Technology, Faculty of Technical Mathematics and Informatics, report no. 98-17, 1998. See also the online version at: http://aw.twi.tudelft.nl/ koekoek/askey/.

15. T.H. KOORNWINDER : Orthogonal polynomials with weight function (1$x)^{\alpha}(1+x)^{\beta}+M \delta(x+1)+N \delta(x-1)$. Canad. Math. Bull. 27(2), 1984, 205-214.

16. K.H. Kwon, L.L. Littlejohn \& G.J. Yoon : Bochner-Krall orthogonal polynomials. Preprint, 1999. 